\title{
Lightly Supervised Discriminative Training of Grapheme Models for Improved Sentence-level Alignment of Speech and Text Data
}

\author{
Adriana Stan ${ }^{1}$, Peter Bell ${ }^{2}$, Junichi Yamagishi ${ }^{2,3}$, Simon King ${ }^{2}$ \\ ${ }^{1}$ Communications Department, Technical University of Cluj-Napoca, Romania \\ ${ }^{2}$ Centre for Speech Technology Research, University of Edinburgh, United Kingdom \\ ${ }^{3}$ National Institute of Informatics, Japan \\ adriana.stan@com.utcluj.ro,Peter.Bell@ed.ac.uk, jyamagis@inf.ed.ac.uk, Simon.King@ed.ac.uk
}

\begin{abstract}
This paper introduces a method for lightly supervised discriminative training using MMI to improve the alignment of speech and text data for use in training HMM-based TTS systems for low-resource languages. In TTS applications, due to the use of long-span contexts, it is important to select training utterances which have wholly correct transcriptions. In a low-resource setting, when using poorly trained grapheme models, we show that the use of MMI discriminative training at the grapheme-level enables us to increase the amount of correctly aligned data by $40 \%$, while maintaining a $7 \%$ sentence error rate and $0.8 \%$ word error rate. We present the procedure for lightly supervised discriminative training with regard to the objective of minimising sentence error rate.
\end{abstract}

Index Terms: automatic alignment, grapheme models, light supervision, MMI, text-to-speech

\section{Introduction}

Recent advances in HMM-based text-to-speech synthesis (TTS) have made the use of large volumes of imperfect, natural speech data an essential aspect in developing systems for new speakers, dialects or even whole languages. When sourcing expressive speech data for system building, we have the choice of recording the speaker under a carefully controlled conditions, or else to manually transcribe and annotate several hours of an existing speech corpus. Neither option is efficient for developers seeking to control the selection of multiple speakers or speaking styles for building synthetic voices.

Another disadvantage of the conventional speech databases is the fact that they are in general recorded as individual utterances, with no correlation in between them or across a paragraph or recording session. To alleviate this problem, the research community has recently shifted focus towards the use of audiobooks as a readily available, more expressive speech resource $[1,2,3,4,5,6,7]$. However, the use of audiobook data, not specifically recorded for speech technology applications, requires a reliable matching transcription to be obtained. The use of an existing automatic speech recognition (ASR) system for this purpose has been proposed by many researchers $[3,8,9,10,11,12,13]$. But these methods are applicable only to languages where the resources for training a good speakerindependent ASR system already exist. For an under-resourced language, the only audio data available may be audiobook data for the target speaker. In this case, a method is required which is able to use only this data, with possibly unreliable transcriptions, with no bootstrapping from other data. Some of these approaches include the detection of specific landmarks in the audio, such as in [14], or the use of TTS systems for speech to speech synchronisation [15]. The latter also requires the existence of a TTS system framework in that language, or at least specific language knowledge.

The approach taken in this study follows from our previous work [16] where we used lightly supervised acoustic model training, somewhat similar to that in [17], using only grapheme models instead of phone models, under the assumption that a manually-created phone dictionary may not be available for an arbitrary new language. This makes the alignment problem harder, since grapheme-based acoustic models (AM) perform worse than phone-based ones [18]. In our earlier work, the success of the method relies on us making a conservative selection of data where the automatically-retrieved transcription is judged to be reliable, using the poor acoustic models.

This paper investigates methods for improving the performance by the use of discriminative training of the grapheme models. Although the use of discriminative training - using, for example, the Maximum Mutual Information (MMI) or Minimum Phone Error (MPE) criterion [19, 20, 21] - is widespread in ASR systems, it is not common in TTS applications, where the generative nature of the HMMs is important. However, methods such as MMI are known to improve on Maximum Likelihood (ML) training where the underpinning model correctness assumption does not hold [22, 23], a particular problem for grapheme-based models. Furthermore, as we discuss in 3.1 the MMI criterion is better matched to the objective of minimising SER - this is important, because in TTS applications, due to the role of long-span contexts in model training, it is more important to obtain utterances which have wholly correct transcriptions [24].

The paper is structured as follows. In Section 2 we briefly present the speech and text alignment method used, following in Section 3 with a description of the acoustic model building procedure, emphasising the advantages of discriminative training in minimising the sentence error rate. Error rate improvements and amount of data aligned using the discriminative training method are then showed and analysed in Section 4. Discussion and conclusions of these results are presented in Sections 5 and 6.

\section{Low-resource alignment of speech and text data}

In our previous study [16], we presented an unsupervised, language independent method for aligning speech data with imperfect transcripts. The method relies solely on the available speech and text resources, and uses a highly restricted word net- 


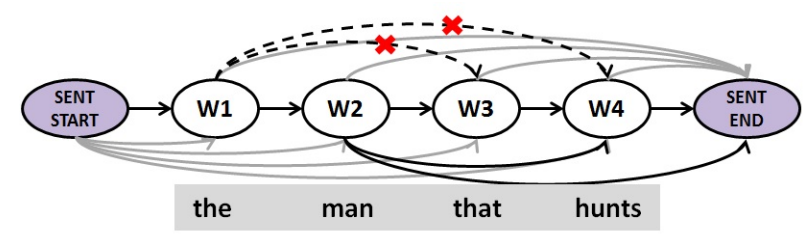

Figure 1: Word skip network design.

work to perform a Viterbi alignment with the poor graphemelevel acoustic models for each segment. This word network, which we term a skip network is built using the available text. Based on the average word duration computed from the speech data, we estimate a broad text window (approx. 2000 words) around the hypothesised utterance transcription. The core principle is to allow the alignment to begin or end at any point in the text window, whilst constraining the word ordering to match word strings in the text, up to a maximum of 2 word deletions or skips. Further, network paths not found in a bigram language model derived from the same text are removed (Fig. 1 illustrates the network). This network imposes much tighter constraints on the output than the traditional biased language model approaches such as [9].

Starting from only 10 minutes of manually transcribed speech from the data to be aligned, we built a first set of poor grapheme-level acoustic models. Using the skip network, we run the acoustic models over the entire speech data. A confidence measure for the aligned data is obtained by comparing the acoustic scores of the recognised output, using different degrees of freedom in the skip network: a network which allows no skips, and a network which allows at most 2 word skips or deletions. The confident data is then used to retrain a new set of acoustic models, and the process repeats. After two iterations, we were able to extract $54.1 \%$ of utterances with high confidence, with the scores of $7.64 \%$ SER and $0.5 \%$ WER on the recovered text.

Some light pre- and post-processing of the data is performed (see Fig. 2): text parts which we suspect the reader omitted, such as the licence agreement and table of contents were discarded; because most of the errors were caused by sentence initial or final short word insertions or deletions, we compared the recognised text with the text-based sentence segmentation, and inserted or deleted the words which did not correspond to it.

\section{Acoustic model training}

\subsection{Discriminative objective functions for SER minimisa- tion}

In conventional maximum likelihood training for HMM-based TTS systems, we aim to find parameters $\theta$ which best explain the training data, in the sense of maximising, over all training utterances, $r$, the joint likelihood of acoustic observations $O_{r}$ and word sequences $W_{r}$. This leads to the objective function

$$
F_{\mathrm{ML}}(\theta)=\sum_{r} \log p_{\theta}\left(O_{r} \mid W_{r}\right) P\left(W_{r}\right):=\sum_{r} D_{\theta}\left(O_{r}, W_{r}\right)
$$

where $D_{\theta}(O, W)=\log p_{\theta}(O \mid W) P(W)$ is a discriminant function. In contrast, discriminative training seeks to explicitly consider the classification decisions made by the resulting model. If the objective is to maximise the number of completely

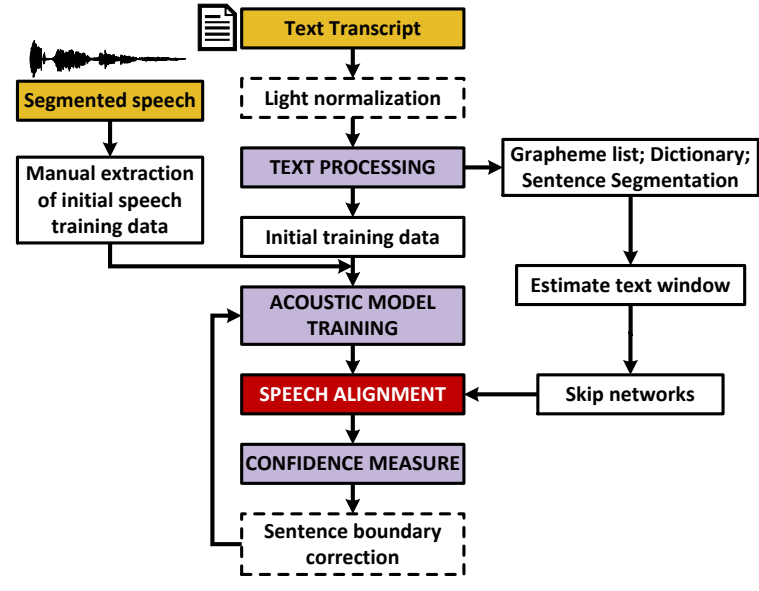

Figure 2: Flowchart of the training and alignment process.

correct sentences, minimising SER, a natural choice of objective function is the Minimum Classification Error (MCE) criterion [25], considering the error on a complete-sentence basis. This function is, however, difficult to optimise. An alternative is to maximise the margin $\mathcal{E}_{r}$ by which utterances are correctly classified,

$$
\mathcal{E}_{r}=D_{\theta}\left(O_{r}, W_{r}\right)-\max _{W} D_{\theta}\left(O_{r}, W\right)
$$

which leads to the objective function

$$
\begin{aligned}
F(\theta) & =\sum_{r} \mathcal{E}_{r} \\
& \geq \sum_{r}\left[D_{\theta}\left(O_{r}, W_{r}\right)-\log \sum_{W} e^{D_{\theta}\left(O_{r}, W\right)}\right] \\
& =\sum_{r} \log \frac{p_{\theta}\left(O_{r} \mid W_{r}\right) P\left(W_{r}\right)}{\sum_{W} p_{\theta}\left(O_{r} \mid W\right) P(W)}=F_{\mathrm{MMI}}(\theta)
\end{aligned}
$$

where we use the softmax approximation to derive a lower bound that is easier to optimise. This is the well-known MMI criterion for discriminative training. It can be shown $[22,26]$ that the expected error rate using MMI-trained models converges to the model-free expected error rate as the amount of training data increases: in other words, MMI does not require the correct generative model to be used in order to be effective. In practice, the acoustic probabilities in Equation 5 are scaled by the inverse of the language model scaling factor; the sum over all words $W$ in the denominator is computed over a lattice.

\subsection{Lightly supervised MMI training}

For the practical application of MMI training in the lowresource, lightly supervised setting, a number of issues must be considered. Firstly, since the MMI objective function is based on the difference between numerator and denominator terms for each utterance $r$, it is important to select only utterances for which we already have a confident text alignment. This problem has been considered by [27]. Secondly, we must consider how the the denominator lattices should be constructed. In ASR applications, a weakened version of the language model used for recognition would be used: for TTS, there is no such model, and for a new language, we may not have good coverage to build one. An alternative is to generate denominator lattices over graphemes, similar to early approaches for discriminative 
training for ASR, such as [28], where phone-level lattices were used.

In this work, the confident utterances were selected using the alignment and confidence measure described in [16] and for which we showed a WER of less than $1 \%$. For denominator lattices instead, we compared the use of both word-level and grapheme-level lattices. Both word and grapheme level training used a bigram language model derived from the original text. But for the grapheme-level lattices, the text was first converted into grapheme sequences. Details of the full implementation are given in Section 3.3.

\subsection{Training procedure}

To test our hypothesis, we built 5 different types of acoustic models starting from the five-state, left-to-right, monographemes with eight mixture components per state, and no state tying.

The first step in building the final acoustic models was to extend the mono-grapheme models to tri-grapheme models, using a standard procedure. The list of tri-graphemes was extracted from the text, so the models will not generalise well to unseen text, but are adequate for aligning the available text. Though to reduce over-fitting, we model only withinword context-this also reduces the number of models. Eight re-estimations were performed, using the confident transcripts of the mono-grapheme ML trained models.

As discussed in Section 3.2, for discriminative training two approaches were selected, one using word-level lattices, the other using grapheme-level ones. For the word-level training, denominator word lattices were built by running the trigrapheme models with a bigram LM derived from the original text, over the training data. Numerator lattices were generated from the approximate transcripts obtained in the alignment process. For the grapheme-level training, the entire text was converted into grapheme sequences and a grapheme language model was built. Numerator and denominator lattices were obtained in a similar way to that presented above. Both word- and grapheme-level training were applied to the monographeme ML models. And only word-level lattices were used to re-estimate the tri-grapheme models.

The generated lattices and the approximate transcriptions were used to find the grapheme model boundaries and produce denominator and numerator model-marked lattices. The MMI models were obtained by re-estimating the ML models over 8 iterations, with a grammar scale factor of 30 . The schematic diagram of the entire AM training process is presented in Fig.3.

\section{Results}

In order to test the lightly supervised discriminative training method, we used an audiobook of A Tramp Abroad by Mark Twain, ${ }^{1}$ for which the GOLD-standard sentence-level segmentation and transcription was kindly provided by Toshiba Reseach Europe Limited, Cambridge Research Laboratory. All SER and WER figures are computed with reference to this transcription.

We first investigated the effect of using poor initial alignments on the effectiveness of MMI training, as well as the quantity of training data. To do this, we applied MMI training to initial ML-trained mono-grapheme models using different versions of the reference transcription. Table 1 presents result with four cases: GOLD denotes the entire audiobook data with the

\footnotetext{
${ }^{1}$ http://librivox.org/a-tramp-abroad-by-mark-twain/
}

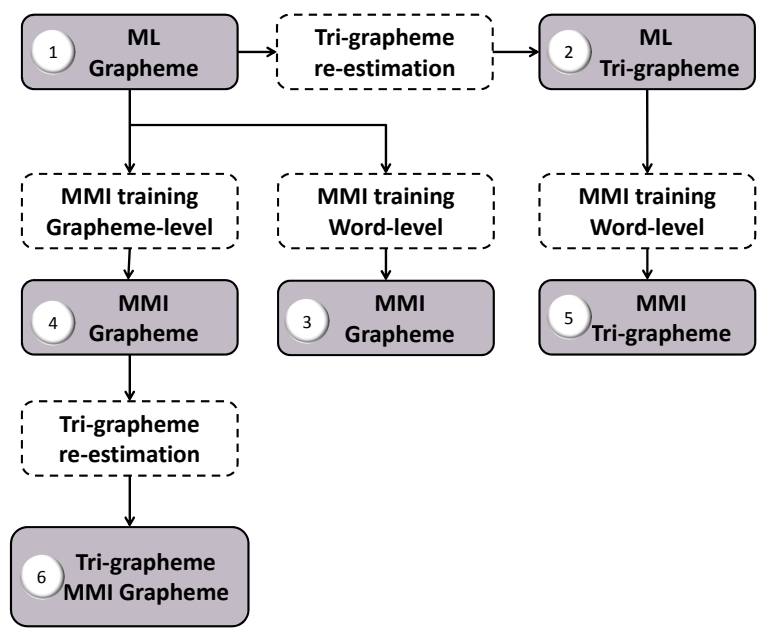

Figure 3: Schematic diagram of the acoustic model training method.

Table 1: The influence of the amount and quality of training data transcription over the accuracy of the grapheme MMI model.

\begin{tabular}{ccc}
\hline Training Data & $\begin{array}{c}\text { SER } \\
{[\%]}\end{array}$ & $\begin{array}{c}\text { WER } \\
{[\%]}\end{array}$ \\
\hline GOLD & 17.62 & 2.29 \\
CONF & 17.40 & 2.24 \\
ALL & 17.80 & 2.26 \\
IT0 & 19.50 & 2.49 \\
\hline
\end{tabular}

ground-truth transcriptions; $\mathrm{CONF}$ is only the confident utterances selected by the alignment procedure using the ML monographeme acoustic model (approx. 54\% of the data); ALL is the entire data with transcriptions obtained using the ML monographeme acoustic model; finally, IT-0 represents the data obtained using the very initial ML acoustic model, which was trained on only 10 minutes of data. ${ }^{2}$ From the results, it may be observed that the MMI training is relatively robust to the use of possibly incorrect transcriptions. Even in the case of IT-0, we find that the use of MMI training results in a SER reduction of $2.5 \%$ and a WER reduction of $0.5 \%$, compared to figures reported in our previous study of $22 \%$ and $3 \%$ respectively.

One other evaluation refers to the use of the tri-grapheme re-estimation and the two different lattice building methods, word and grapheme-level lattices. The 5 acoustic models are evaluated from a SER and WER point-of-view, and the results are presented in Table 2. The numerator lattices are built from the confident data obtained with the baseline AM. Acoustic model descriptions are as follows: ${ }^{3}$ (1) ML-MG - baseline ML trained mono-grapheme acoustic models; (2) ML-TG ML trained tri-grapheme acoustic models; (3) MMI-MG-WD - MMI trained mono-grapheme AM using word-level lattices; ( 4 ) MMI-MG-GR - MMI trained mono-grapheme AM using grapheme-level lattices; (4) MMI-TG - MMI trained trigrapheme AM with word-level lattices; (5) TG-MMI-MG MMI trained mono-grapheme AM with grapheme-level lattices and then re-estimated into tri-grapheme models.

\footnotetext{
${ }^{2}$ See [16] for the initial acoustic model description

${ }^{3}$ Numbers correspond to those in Fig. 3
} 
Table 2: SER and WER for the entire data using different acoustic models and the confident utterances as training data. Reference is the gold-standard transcript.

\begin{tabular}{lcc}
\hline Acoustic Model & $\begin{array}{c}\text { SER } \\
{[\%]}\end{array}$ & $\begin{array}{c}\text { WER } \\
{[\%]}\end{array}$ \\
\hline (1) ML-MG & 18.72 & 2.43 \\
(2) ML-TG & 17.44 & 5.88 \\
(3) MMI-MG-WD & 17.62 & 2.25 \\
(4) MMI-MG-GR & 17.40 & 2.24 \\
(5) MMI-TG-WD & 14.98 & 3.59 \\
(6) TG-MMI-MG & 12.15 & 1.84 \\
\hline
\end{tabular}

Table 3: The percentage of confident data and the error rates for each acoustic model. SER and WER are computed against the gold-standard transcript.

\begin{tabular}{lccc}
\hline Acoustic Model & $\begin{array}{c}\text { Percent } \\
{[\%]}\end{array}$ & $\begin{array}{c}\text { SER } \\
{[\%]}\end{array}$ & $\begin{array}{c}\text { WER } \\
{[\%]}\end{array}$ \\
\hline (1) ML-MG & 54.10 & 7.64 & 0.5 \\
(2) ML-TG & 70.11 & 8.29 & 1.32 \\
(3) MMI-MG-WD & 57.01 & 9.36 & 0.61 \\
(4) MMI-MG-GR & 59.29 & 9.85 & 0.57 \\
(5) MMI-TG-WD & 70.37 & 7.54 & 0.87 \\
(6) TG-MMI-MG & 75.88 & 7.59 & 0.80 \\
\hline
\end{tabular}

Although the error rates for the tri-grapheme reestimated models (ML-TG), and the grapheme-level lattices (MMI-MG-GR) do not show a significant decrease for the overall SER and WER, we will show that their advantages are important when determining the confident amount of data aligned. However, when doing both word-level training and tri-grapheme re-estimation (TG-MMI-MG) going from baseline mono-grapheme (ML-MG) models, the overall improvement is of $6.5 \%$ in SER and $0.6 \%$ in WER. This means that even with the poor acoustic models, this method manages to correctly align almost $90 \%$ of the original data. However, the final results are also influenced by the choice of confidence measure [29].

Note that the most important goal of the whole process is to be able to extract greater quantities of data from the speech and text resources available. Considering this aim, Table 3 presents the proportion of confident alignments obtained by each of the above acoustic models, along with their SER and WER. The acoustic model description is as above. Observe that simply by moving from the ML mono-grapheme models (ML-MG) to ML tri-grapheme models ( $M L-T G)$, the number of confident alignments increases by $30 \%$, going from $54.1 \%$ to $70.1 \%$ while maintaining approximately the same error rates. Results also show that grapheme-level lattices improve the confidently aligned percentage by about $2 \%$ when comparing MMI-MG-GR and MMI-MG-WD, and that word-level discriminative training over tri-grapheme models, brings no relative improvement.

The best performing model, as expected, is the trigrapheme derived from the MMI-trained mono-graphemes which results in an increase of $40 \%$ in the quantity of confident data for the same levels of error. These results are comparable in WER to those presented in [9], where good acoustic and language models are used to align the data.

\section{Discussion}

Unsupervised or lightly supervised speech and text alignment is an important prerequisite in the process of building text-tospeech systems in a language or domain where language analysis and acoustic model building are difficult or expensive (i.e. annotated speech resources are scarce and there is little or no language expertise).

The method presented here, however, showed that using only speech data and its approximate orthographic transcript, almost $70 \%$ can be aligned ${ }^{4}$. The improvements obtained by both the tri-grapheme and lightly supervised acoustic models are significant, and amount to an overall increase of $40 \%$ while maintaining the word and sentence error rates. In a separate study, we obtained similar numbers for 14 other resources in 14 different languages [30]. Also, [31] showed no definite preference towards TTS voices built on manually aligned data, as opposed to our ML approach. Therefore by increasing the amount of confident data using tri-graphemes and discriminative training, the synthesis quality can only improve.

The major goal of this ongoing work is to be able to align the entire speech resource available by taking into account audio insertions and substitutions as well. The confidence measure may also be improved, so that the error rates of the confident files are close to $0 \%$. Currently, the difference between the computed accuracy of the models and the percentage of confident files emphasizes the need for a better confidence measure. One other issue which might occur in found data is the different recording conditions across sections or chapters of the speech resource. A way to overcome the potential alignment problems would be to use multi-condition training [32, 33].

\section{Conclusions}

This paper introduced a series of enhancements for poor grapheme acoustic models used in the alignment of speech and text data. Extending the mono-grapheme models to tri-graphemes and then performing discriminative training at grapheme-level increased the percent of extracted confident data by $40 \%$ in the same SER and WER conditions. Compared to other studies on limited domain recognition, our methods are based only on the available data and use minimal user intervention and language expertise.

\section{Acknowledgements}

The research leading to these results has received funding from the European Community's Seventh Framework Programme (FP7/2007-2013) under grant agreement no. 287678. (Simple4All).

The GOLD transcripts for A Tramp Abroad were very kindly provided by Toshiba Research Europe Limited, Cambridge Research Laboratory. The grapheme-based acoustic model training tools were provided by Oliver Watts.

\footnotetext{
${ }^{4}$ Confident percentage is $75 \%$, but taking into account the $7 \%$ SER, we are left with $70 \%$ fully correct data
} 


\section{References}

[1] L. Chen, M. J. F. Gales, V. Wan, J. Latorre, and M. Akamine, "Exploring Rich Expressive Information from Audiobook Data Using Cluster Adaptive Training," in Proc. of Interspeech, 2012.

[2] É. Székely, J. P. Cabral, M. Abou-Zleikha, P. Cahill, and J. Carson-Berndsen, "Evaluating expressive speech synthesis from audiobook corpora for conversational phrases," in Proc. of LREC, 2012, pp. 3335-3339.

[3] É. Székely, T. G. Csapó, B. Tóth, P. Mihajlik, and J. CarsonBerndsen, "Synthesizing expressive speech from amateur audiobook recordings," in Proc. IEEE Workshop on Spoken Language Technology, Miami, Florida, USA, 2012, pp. 297-302.

[4] K. Prahallad and A. W. Black, "Segmentation of Monologues in Audio Books for Building Synthetic Voices," IEEE Transactions on Audio, Speech \& Language Processing, vol. 19, no. 5, pp. 1444-1449, 2011

[5] N. Braunschweiler and S. Buchholz, "Automatic Sentence Selection from Speech Corpora Including Diverse Speech for Improved HMM-TTS Synthesis Quality," in Proc. of Interspeech, 2011, pp. 1821-1824.

[6] É. Székely, J. P. Cabral, P. Cahill, and J. Carson-Berndsen, "Clustering Expressive Speech Styles in Audiobooks Using Glotta Source Parameters," in Proc. of Interspeech, Florence, Italy, Aug. 2011, pp. 1821-1824.

[7] M. Paulik and P. Panchapagesan, "Leveraging large amounts of loosely transcribed corporate videos for acoustic model training," in Proc. of ASRU, 2011, pp. 95-100.

[8] O. Boeffard, L. Charonnat, S. L. Maguer, and D. Lolive, "Towards Fully Automatic Annotation of Audio Books for TTS," in Proc. of LREC, Istanbul, Turkey, May 2012.

[9] N. Braunschweiler, M. Gales, and S. Buchholz, "Lightly supervised recognition for automatic alignment of large coherent speech recordings," in Proc. of Interspeech, 2010, pp. 2222-2225.

[10] K. Prahallad, A. R. Toth, and A. W. Black, "Automatic building of synthetic voices from large multi-paragraph speech databases," in Proc. of Interspeech, 2007, pp. 2901-2904.

[11] P. Moreno and C. Alberti, "A factor automaton approach for the forced alignment of long speech recordings," in Proc. of ICASSP, 2009, pp. 4869-4872.

[12] G. Bordel, M. Peñagarikano, L. J. Rodríguez-Fuentes, and A. Varona, "A simple and efficient method to align very long speech signals to acoustically imperfect transcriptions," in Proc of Interspeech, 2012.

[13] M. Alessandrini, G. Biagetti, A. Curzi, and C. Turchetti, "SemiAutomatic Acoustic Model Generation from Large Unsynchronized Audio and Text Chunks," in Proc. of Interspeech, 2011, pp. 1681-1684.

[14] A. Haubold and J. Kender, "Alignment of Speech to Highly Imperfect Text Transcriptions," CoRR, vol. abs/cs/0612139, 2006.

[15] X. Anguera, N. Perez, A. Urruela, and N. Oliver, "Automatic synchronization of electronic and audio books via TTS alignment and silence filtering," in Proc of ICME, 2011, pp. 1-6.

[16] A. Stan, P. Bell, and S. King, "A Grapheme-based Method for Automatic Alignment of Speech and Text Data," in Proc. of IEEE Workshop on Spoken Language Technology, Miami, Florida, USA, Dec. 2012, pp. 286-290.

[17] L. Lamel, J.-L. Gauvain, and G. Adda, "Lightly Supervised and Unsupervised Acoustic Model Training," Computer Speech and Language, vol. 16, pp. 115-129, 2002.

[18] M. Killer, S. Stüker, and T. Schultz, "Grapheme based speech recognition," in Proc. of Eurospeech, 2003, pp. 3141-3144.

[19] M. J. F. Gales, "Discriminative models for speech recognition," in Information Theory and Applications Workshop, 1997, pp. 2-3

[20] G. Heigold, H. Ney, R. Schluter, and S. Wiesler, "Discriminative Training for Automatic Speech Recognition: Modeling, Criteria, Optimization, Implementation, and Performance," Signal Processing Magazine, IEEE, vol. 29, no. 6, pp. 58-69, Nov. 2012.
[21] J. L. Roux and E. McDermott, "Optimization methods for discriminative training," in Proc. of Interspeech, 2005, pp. 33413344.

[22] R. Schlüter and H. Ney, "Model-based MCE bound to the true Bayes' error," IEEE Signal Processing Letters, vol. 8, no. 5, 2001.

[23] L. Mathias, G. Yegnanarayanan, and J. Fritsch, "Discriminative Training of Acoustic Models Applied to Domains with Unreliable Transcripts," in Proc. of ICASSP, vol. 1, 2005, pp. 109-112.

[24] J. Ni and H. Kawai, "An Investigation of the Impact of Speech Transcript Errors on HMM Voices," in Proc. of 7th ISCA Workshop on Speech Synthesis, 2010, pp. 246-251.

[25] B. H. Juang and S. Katagiri, "Discriminative Learning for Minimum Error Classification," IEEE Transactions on Signal Processing, vol. 40, no. 12, pp. 3043-3053, 1992.

[26] G. Bouchard and B. Triggs, "The trade-off between generative and discriminative classifiers," in Proceedings of 16th Symposium of IASC Computational Statistics, 2004, pp. 721-728.

[27] H. Chan and P. Woodland, "Improving broadcast news transcription by lightly supervised discriminative training," in Proc. of ICASSP, vol. 1, may 2004, pp. 737-740.

[28] J. Zheng and A. Stolcke, "Improved discriminative training using phone lattices," in Proc. of Interspeech, 2005, pp. 2125-2128.

[29] H. Jiang, "Confidence measures for speech recognition: A survey," Speech Communication, vol. 45, no. 4, pp. 455-470, Apr. 2005.

[30] A. Stan, O. Watts, Y. Mamiya, M. Giurgiu, R. Clark, J. Yamagishi, and S. King, "TUNDRA: A Multilingual Corpus of Found Data for TTS Research Created with Light Supervision," in Proc. of Interspeech, 2013.

[31] Y. Mamiya, J. Yamagishi, O. Watts, R. Clark, S. King, and A. Stan, "Lightly Supervised GMM VAD to use Audiobook for Speech Synthesiser," in Proc. of ICASSP, 2013.

[32] O. Kalinli, M. Seltzer, J. Droppo, and A. Acero, "Noise Adaptive Training for Robust Automatic Speech Recognition," Audio, Speech, and Language Processing, IEEE Transactions on, vol. 18, no. 8, pp. 1889-1901, 2010.

[33] H. Liao and M. J. F. Gales, "Adaptive training with joint uncertainty decoding for robust recognition of noisy data," in Proc. of ICASSP, 2007, pp. 389-392. 